\title{
Ureter Part
}

National Cancer Institute

\section{Source}

National Cancer Institute. Ureter Part. NCI Thesaurus. Code C160613.

A named segment of the ureter. 